\title{
Migration arrest of chemoresistant leukemia cells mediated by MRTF-SRF pathway
}

\author{
Maho Morimatsu1, Erika Yamashita 1,2,3*, Shigeto Seno ${ }^{4}$, Takao Sudo ${ }^{1,2,3}$, Junichi Kikuta, ${ }^{1,2,3}$, Hiroki Mizuno ${ }^{1,2}$,
} Daisuke Okuzaki ${ }^{2,5}$, Daisuke Motooka ${ }^{5}$ and Masaru Ishii ${ }^{1,2,3^{*}}$

\begin{abstract}
Background: Dormant chemotherapy-resistant leukemia cells can survive for an extended period before relapse. Nevertheless, the mechanisms underlying the development of chemoresistance in vivo remain unclear.

Methods: Using intravital bone imaging, we characterized the behavior of murine acute myeloid leukemia (AML) cells (C1498) in the bone marrow before and after chemotherapy with cytarabine.

Results: Proliferative C1498 cells exhibited high motility in the bone marrow. Cytarabine treatment impaired the motility of residual C1498 cells. However, C1498 cells regained their migration potential after relapse. RNA sequencing revealed that cytarabine treatment promoted MRTF-SRF pathway activation. MRTF inhibition using CCG203971 augmented the anti-tumor effects of chemotherapy in our AML mouse model, as well as suppressed the migration of chemoresistant C1498 cells.

Conclusions: These results provide novel insight into the role of cell migration arrest on the development of chemoresistance in AML, as well as provide a strong rationale for the modulation of cellular motility as a therapeutic target for refractory AML.
\end{abstract}

Keywords: In vivo imaging, Microscopic imaging, Leukemia, Bone marrow, Cell migration, Cell motility, Chemoresistance, Cancer therapy, Serum response factor (SRF)

\section{Background}

Chemotherapy with metabolic antagonists, such as cytarabine (cytosine arabinoside), has been widely used for the treatment of acute myeloid leukemia (AML) for more than 40 years [1-3]. Nevertheless, even high doses of cytarabine result in disease remission in only $40 \%$ of AML patients due to the development of chemoresistance $[4,5]$. Chemoresistant AML cells interact with various cell types in the bone marrow (BM), including endothelial cells, osteoblasts, and stromal cells, and these interactions are believed to play essential roles in the development of

\footnotetext{
*Correspondence: erika-ys@icb.med.osaka-u.ac.jp; mishii@icb.med.osakau.ac.jp

${ }^{1}$ Department of Immunology and Cell Biology, Graduate School of Medicine and Frontier Biosciences, Osaka University, Osaka, Japan

Full list of author information is available at the end of the article
}

resistance [6]. Furthermore, compared to $\mathrm{T}$ cell acute leukemia cells, AML cells interacted extensively with the BM microenvironment in an MLL-AF9-driven AML mouse model $[7,8]$. In vivo studies led to the identification of several candidate molecules with important roles in the interaction between AML cells and BM microenvironment components [9-13].

On the other hand, another study suggested that AML cell migration did not depend on interactions with ligands found in the BM stroma [7]. These contradictive findings highlight the complexity of the mechanisms underlying the interactions between AML cells and BM microenvironment components. The identification of the key BM components that regulate AML chemoresistance remains a significant unmet need in the treatment of AML. Moreover, characterization of the exact localization and

(c) The Author(s). 2020 Open Access This article is licensed under a Creative Commons Attribution 4.0 International License, which permits use, sharing, adaptation, distribution and reproduction in any medium or format, as long as you give appropriate credit to the original author(s) and the source, provide a link to the Creative Commons licence, and indicate if changes were made. The images or other third party material in this article are included in the article's Creative Commons licence, unless indicated otherwise in a credit line to the material. If material is not included in the article's Creative Commons licence and your intended use is not permitted by statutory regulation or exceeds the permitted use, you will need to obtain permission directly from the copyright holder. To view a copy of this licence, visit http://creativecommons.org/licenses/by/4.0/ 
movements of chemotherapy-resistant AML cells within the $\mathrm{BM}$ is of high importance for a better understanding of the mechanisms underlying resistance.

Herein, we provide further insight into the behavior of chemoresistant AML cells in vivo with evidence from intravital time-lapse bone imaging. We further identify the key molecular mechanisms of migration arrest and chemoresistance development in AML cells.

\section{Methods}

\section{Mice}

C57BL/6 J mice were purchased from Crea Japan Inc. (Tokyo, Japan). Female mice (8-10 weeks of age) were used unless stated otherwise. The number of animals used per group in each experiment is provided in the corresponding figure legend. All mice were maintained under specific pathogen-free conditions, and the Institutional Animal Care and Use Committee of Osaka University approved all procedures involving animals.

\section{Cells}

C1498 cells were purchased from American Type Culture Collection. The green fluorescent protein (GFP) coding sequence was cloned in a pMYs retroviral vector (Cell Biolabs, Inc.). The recombinant virus was produced using the Platinum-E retroviral packaging cell line, Ecotropic (Cell Biolabs, Inc.), which was transfected using PEI MAX (Polysciences, Inc.). C1498 cells were stably transduced using the recombinant retrovirus, grown in DMEM (no glucose or sodium pyruvate, with L-glutamine; Nakalai Tesque, Inc.) supplemented with $10 \%$ fetal bovine serum (FBS). Successfully transduced cells were selected by cell sorting and expanded as single-cell clones. One clone was selected for each transfected cell line (C1498-EGFP). C1498-EGFP cells were maintained in DMEM supplemented with $10 \% \mathrm{FBS}$ and $1 \%$ penicillin-streptomycin at $37^{\circ} \mathrm{C}$ in a humidified atmosphere containing $5 \% \mathrm{CO}_{2}$.

\section{Animal models}

The AML syngeneic mouse model was generated by intravenously injecting $2.0 \times 10^{6}$ C1498-EGFP cells resuspended in $100 \mu \mathrm{L}$ of phosphate-buffered saline (PBS) in C57BL/6 J mice.

\section{Treatment of mice}

AML mice were treated with high-dose cytarabine chemotherapy. Twenty micrograms of cytarabine (Tokyo Chemical Industry Co., Ltd.) dissolved in $200 \mu \mathrm{L}$ PBS was injected intraperitoneally at days 19 and 20 after C1498EGFP cell injection for intravital imaging; for survival analysis, chemotherapy was administered at days 14 and 15 . In addition to cytarabine, some mice also received CCG203971. For imaging, $200 \mu \mathrm{g}$ of CCG-203971 dissolved in $50 \mu \mathrm{L}$ DMSO solution was intraperitoneally injected at day 18, and twice per day at days 19 and 20. For survival curve analysis, $200 \mu \mathrm{g}$ of CCG dissolved in $50 \mu \mathrm{L}$ DMSO was administered at day 13, and twice per day at days 14 and 15.

\section{Intravital imaging}

Intravital two-photon imaging was performed as previously described [14-17]. Imaging was performed using a Nikon upright two-photon microscope (A1RMP) and a laser (Chameleon Vision II Ti: Sapphire; Coherent) at $880 \mathrm{~nm}$; for imaging, a $\times 25$ water immersion objective (APO, N.A. 1.1; Nikon, Tokyo, Japan) was used. Raw images were processed to construct 3D images and maximum intensity projections (MIP) using the Imaris software v9.2.1 (Bitplane). Detection of bone tissues was achieved by second harmonic generation (SHG). And blood vessels were visualized by intravenously injecting Isolectin GS-IB4 from Griffonia simplicifolia, Alexa Fluor ${ }^{\circ} 594$ conjugate $(50 \mu \mathrm{g} / \mathrm{mL}$; Thermo Fisher).

\section{Image quantification}

The distance between the AML cells and the bone or blood vessels was analyzed using the Imaris software. AML cells were defined as spots with a diameter of $10 \mu \mathrm{m}$ using the 3D images. The surface of the bones and blood vessels was identified in the same $3 \mathrm{D}$ image and transformed to distance gradients. Subsequently, the intensities of the distance gradients of the spots were calculated. We analyzed the displacement area of AML cells by calculating IoUs (intersection over union). Initially, all frames (green channels) of MIP images from each experiment were combined, and thresholds were calculated by the Otsu method. These thresholds were used to distinguish cellular regions from the background. Denoising of the resulting binary images was performed by applying median filters. Finally, we calculated the IoUs between the cell regions of successive frames. To calculate the mean migration speed, we defined AML cells as estimated spots with a diameter of $10 \mu \mathrm{m}$ from the MIP image; the spot trajectories were automatically analyzed.

\section{Sorting AML cells}

First bone marrow cells were collected from femur, tibia, and pelvis from three untreated and three cytarabinetreated mice 21 days after AML cell injection. The bone marrow cells were blocked with anti-CD16/32 antibody (553141; BD Biosciences) for $5 \mathrm{~min}$ and stained with PE conjugated anti-CD49b antibody (553858; BD Biosciences) for $30 \mathrm{~min}$. $\mathrm{GFP}^{+} \mathrm{CD}_{4} 9 \mathrm{~b}^{+}$cells were isolated as AML cells using an SH800 cell sorter (Sony).

\section{RNA-Seq}

Total RNA was extracted using QIAzol lysis reagent (Qiagen) according to the manufacturer's instructions. 
Sequencing was performed on an Illumina HiSeq 2500 platform (Illumina) in a 75-base single-end mode and the Illumina Casava software (v1.8.2; Illumina) was used for base calling. Sequenced reads were mapped to mouse reference genome sequences $(\mathrm{mm} 10)$ using TopHat (v2.0.13) in combination with Bowtie2 (v2.2.3) and SAMtools (v0.1.19). The fragments per kilobase of exon per million mapped fragments were calculated using Cufflinks (v2.2.1). Upstream regulator analysis and causal network analysis was performed using Ingenuity Pathway Analysis (IPA; Qiagen) to identify molecular networks within the Ingenuity Knowledge Database. $P$ values (threshold of 0.05 ) and $\mathrm{z}$-scores were used to identify significant upstream regulators. $P$ value indicated significance, while $\mathrm{z}$-scores were used to define activation ( $\mathrm{z}$-score $\geq 2.0$ ) or inhibition ( $\mathrm{z}$-score $\leq-2.0$ ). Access to raw data concerning this study was submitted under Gene Expression Omnibus (GEO) accession number GSE149853.

\section{Statistical analysis}

Numerical data are shown as a dot plot. Data are expressed as means \pm SEM. Statistical significance between groups was determined using two-tailed $t$ tests. One-way analysis of variance (ANOVA) was used for comparisons among three groups, while KolmogorovSmirnov test was used for comparisons between two groups. Fisher's exact test was used to calculate $P$ values in IPA upstream analysis. Statistical significance in survival data was determined using the log-rank test. All the statistical analyses (except for RNA-Seq data) were performed using GraphPad Prism 7 (GraphPad Software).

\section{Results}

\section{Cytarabine treatment promotes transient AML cell} motility reduction

To establish an AML syngeneic mouse model, we transplanted C1498 murine AML cells intravenously into wild-type C57BL/6 J mice [14, 15]. Prior to cell transplantation, C1498 cells were fluorescently labeled with GFP by retroviral transduction, allowing for tracking of the engrafted AML cells. The majority of the mice died between 25 and 30 days after AML cell transfer (Fig. S2); hence, we stratified the disease progression stages into early phase (7-13 days after transplantation), middle phase (14-20 days after transplantation), and late phase (day 21 until death).

Intravital imaging of the parietal BM revealed a constant movement of AML cells along the blood vessels during all disease progression stages (Fig. S1; Video 1). We hypothesized that the development of chemoresistance in AML cells is accompanied by changes in cell motility; thus, we analyzed the dynamics of chemoresistant AML cells in the BM following cytarabine treatment. We administrated high-dose cytarabine $(20 \mu \mathrm{g}$ in $200 \mu \mathrm{L}$ PBS $)$ twice at days 19 and 20 after AML cell transfer; high-dose cytarabine treatment significantly prolonged median survival, and the number of AML cells were significantly decreased (Fig. S2). Although the number of AML cells in the BM transiently decreased in response to cytarabine treatment at day 21, between days 21 and 28, the number of AML cells in the BM increased (Fig. 1a), suggesting AML cell growth.

To compare the dynamics of AML cells between the cytoreductive and proliferative phases after cytarabine treatment, we performed intravital time-lapse imaging on days 21 and 28 (Video 2). AML cell migration is characterized by amoeboid movements, which are driven by repetitive cycles of protrusion and contraction. Thus, we classified AML migration into two types: a constantly changing cell shape characterized by protrusions or migration driven by extensive contraction and protrusion. To quantify the extent of cell shape alterations, we calculated the "Intersection over Union (IoU)" of AML cells using time-lapse imaging data (Fig. 1b). The value of "1 minus IoU" decreased during the cytoreductive phase (Fig. 1c), suggesting reduced protrusion activity. By contrast, protrusion activity increased during the proliferative phase (Fig. 1c). The centroid tracking of individual AML cells revealed that the mean speed of AML cells during the cytoreductive phase was $<1 \mu \mathrm{m} / \mathrm{min}$, and was significantly lower compared with the mean speed of AML cells during the proliferative phase or the control group $(>1 \mu \mathrm{m} / \mathrm{min}$; Fig. 1d, e). These results suggest that cytarabine treatment induces AML cell motility changes likely by modulating the contractile machinery of the cells.

\section{Chemotherapy does not affect AML cell localization in the BM}

Although AML chemoresistance has been reported to rely on the interaction with components of the BM microenvironment, it remains unclear whether AML cell localization within the BM can induce chemoresistance. To determine the localization of chemoresistant AML cells within the BM, we performed three-dimensional (3D) imaging using two-photon microscopy using mice injected with AML cells and subsequently treated with high-dose cytarabine at days 19 and 20 after AML cell transfer. After constructing the pseudo-surface of blood vessels and bones using the Imaris software, we calculated the distance from each AML cell to the nearest blood vessel and bone surface (Fig. 2a). More than 50\% of AML cells were localized within $15 \mu \mathrm{m}$ of bone surfaces in untreated control and cytarabine-treated mice (Fig. 2b, c). Similarly, more than $60 \%$ of AML cells were localized within $5 \mu \mathrm{m}$ of blood vessel surfaces in control and cytarabine-treated mice (Fig. 2d, e). These results suggest that there is no association between AML cell chemoresistance and cell localization in the BM. 


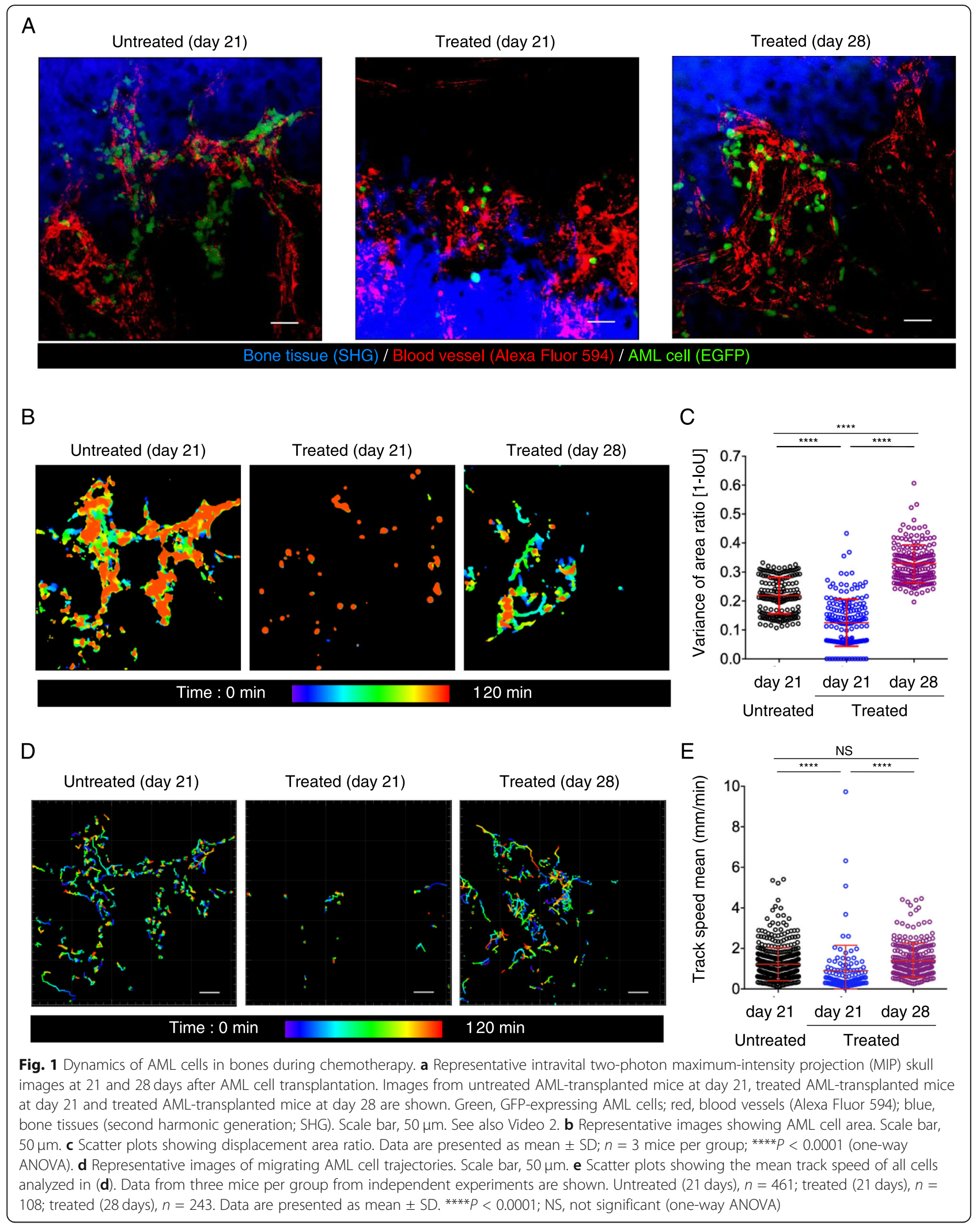


A
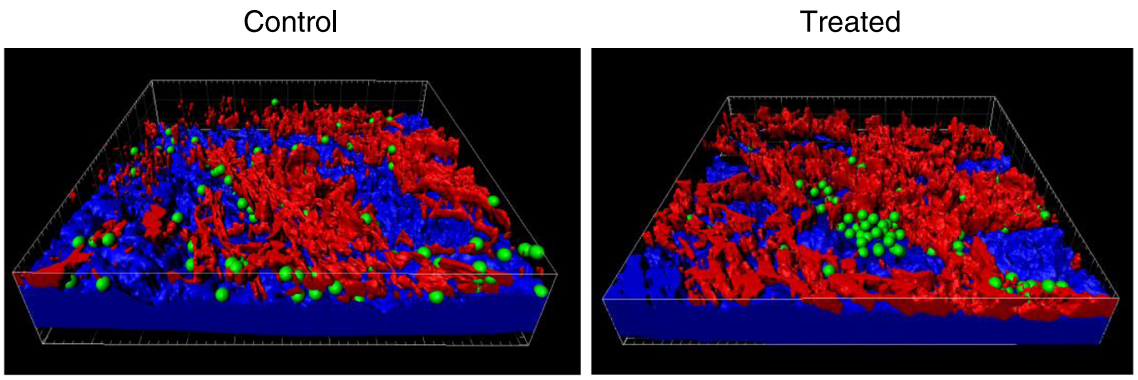

Bone surface / Blood vessel surface / AML cell

B

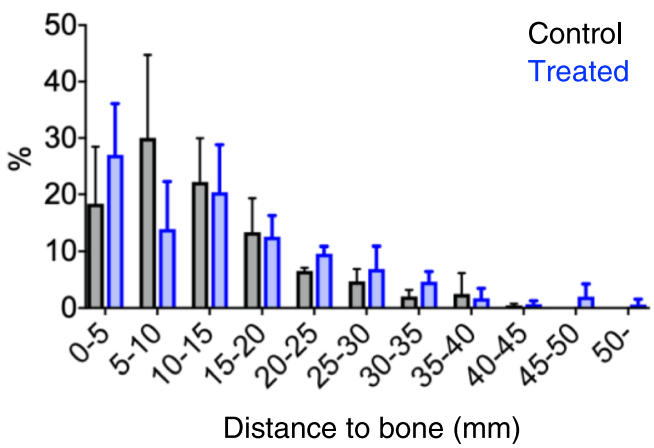

D

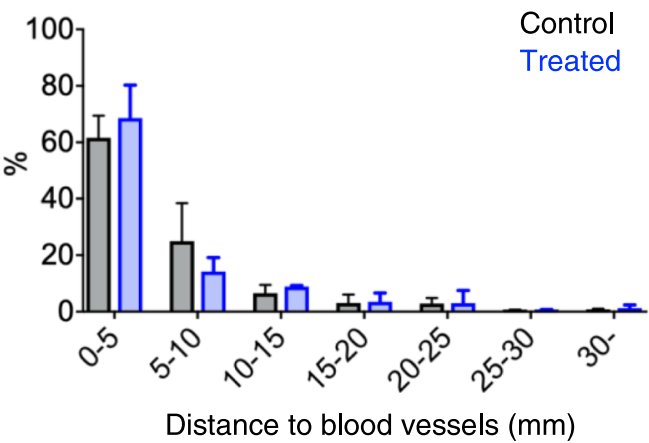

C

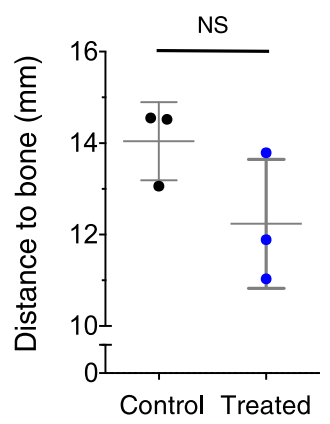

E

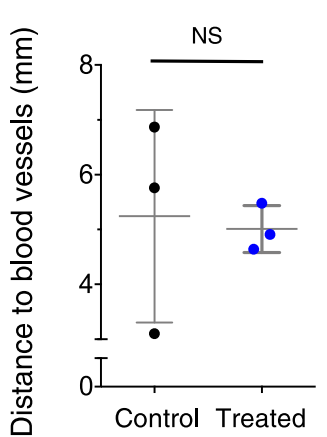

Fig. 2 Localization of chemoresistant AML cells in the bone. a Representative intravital two-photon 3D skull images of AML control mice and AML mice treated with chemotherapy. Green spots, AML cells; red, surface of blood vessels; blue, surface of bone tissues. See also Video 2. b, d Distribution of distance between AML cells and the bone surface (b) or blood vessels (d). Pooled data from three mice per group are shown. Control, $n=400$; treated, $n=235$. c, e Mean distance between AML cells and the bone surface (c) or blood vessels (e). NS, not significant (Kolmogorov-Smirnov test)

\section{Inhibition of MRTF-SRF signaling reverses}

\section{chemoresistance and cell motility changes in AML cells}

To further characterize the chemoresistant AML cells, we analyzed the gene expression profiles of AML cells from cytarabine-treated mice 21 days after AML cell transfer by RNA sequence. Enrichment analysis for diseases and biological functions using the Ingenuity Pathway Analysis (IPA) software revealed that the biological functional categories "cellular movement" and "immune cell trafficking" were significantly suppressed in cytarabine-treated AML cells (Fig. S3A, B). This transcriptional profile was consistent with our intravital imaging results (Video 2). To identify upstream regulators that determine AML cell chemoresistance, we conducted upstream analysis and causal network analysis using IPA. Serum response factor (SRF) and the myocardin-related transcription factors MRTFA (MKL1) and MRTFB (MKL2) were the top three upstream regulators (Fig. 3a). Using IPA network analysis, we found that extensive interactions exist between these three regulators (Fig. 3b). Additionally, DIAP is the actin nucleation and elongation factor that drives serum-dependent SRFMRTFA, as well as several Rho guanine nucleotide exchange factors, were identified as active master regulators in cytarabine-treated AML cells (Fig. S3C). Recent studies 


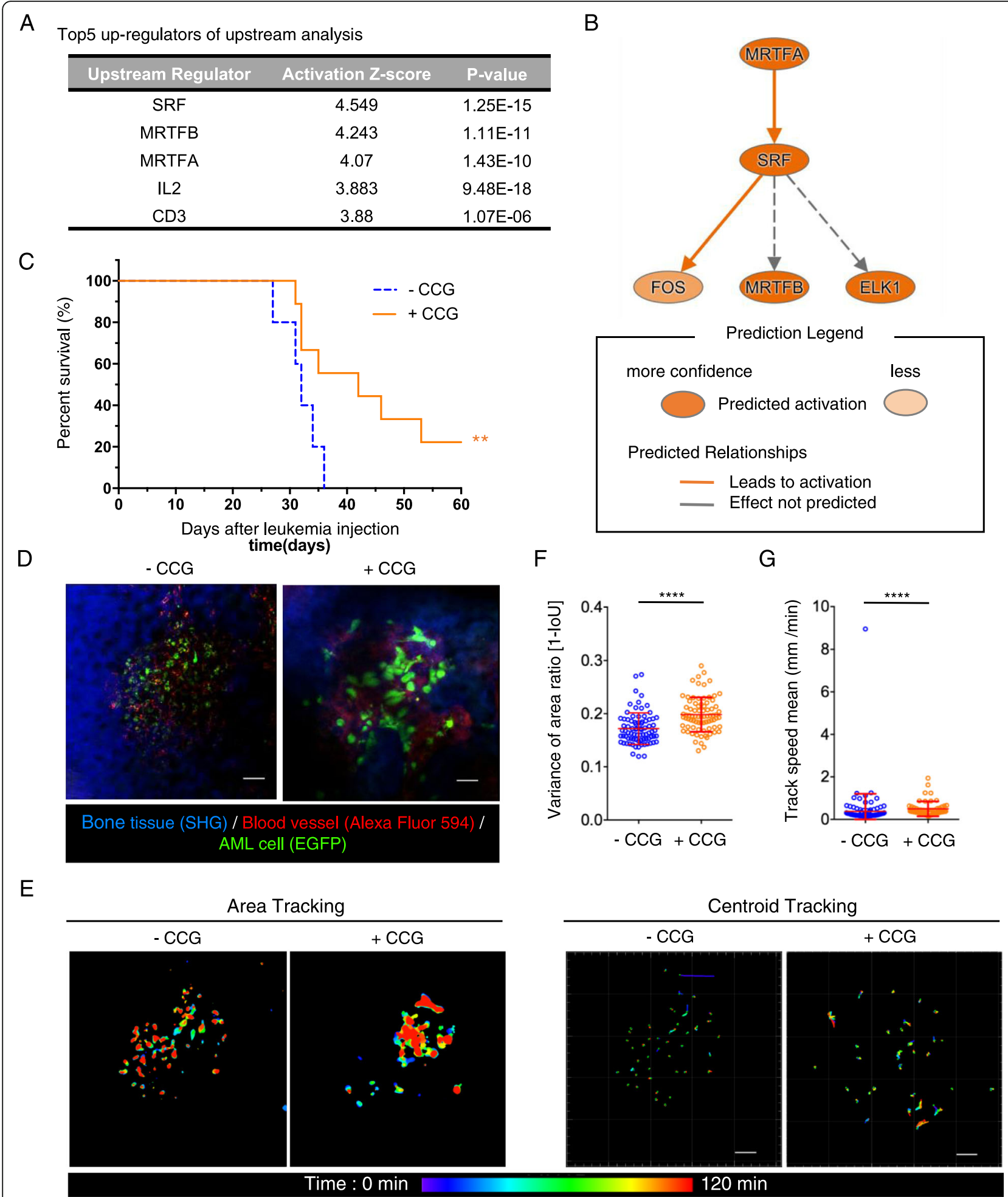

Fig. 3 (See legend on next page.) 


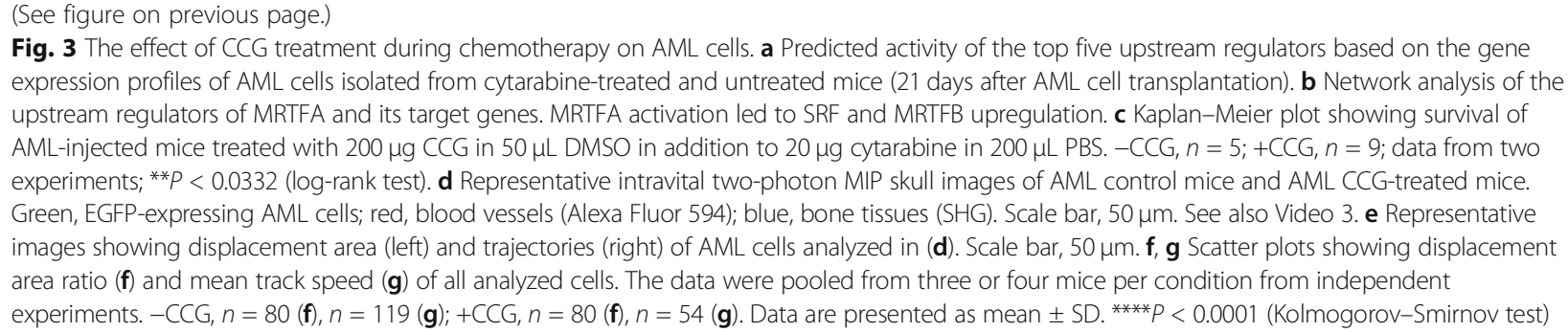

showed that activation of MRTF and SRF is Rhodependent. Moreover, MRTF and SRF have been implicated in cancer cell motility regulation by modulating the construction of actin filament networks [18-20].

To further investigate the role of Rho-MRTF-SRF pathway activation in AML cell motility following cytarabine treatment, we used CCG-203971 [21], a CCG-1423 analog that inhibits Rho-induced SRF-mediated transcription [22]. CCG-203971 (200 $\mu \mathrm{g}$ in $50 \mu \mathrm{L}$ DMSO) was administered five times to cytarabine-treated mice. Kaplan-Meier survival analysis revealed that CCG treatment significantly prolonged survival (Fig. 3c), suggesting that Rho-MRTFSRF inhibition augments the anti-tumor effects of cytarabine. We also assessed the effects of CCG treatment on the localization of chemoresistant AML cells using 3D imaging and surface distance analysis. We found that CCG treatment did not affect the distance between AML cells and blood vessels or bone surfaces (Fig. S4A-D). We also investigated the effect of CCG treatment on AML cell motility and found that CCG treatment promoted continuous cell morphology alterations (Fig. 3d, f; Video 3). The centroid tracking of individual AML cells revealed that the mean speed of AML cells was significantly higher in CCG-treated mice compared with control mice. However, the actual mean speed in CCG-treated mice was less than $1 \mu \mathrm{m} / \mathrm{min}$, similar to that in the control group (Fig. 3e, g). Considering that the average diameter of AML cells is $10 \mu \mathrm{m}$, migration with a speed of less than $1 \mu \mathrm{m} / \mathrm{min}$ can be considered passive migration. Rapidly migrating AML cells were rarely observed in our imaging data (Video 3). Hence, Rho-MRTFSRF inhibition following cytarabine treatment enhanced AML cell protrusion but not contraction. Considering the critical role of the MRTF-SRF pathway in the survival of chemoresistant AML cells, the combination of cytarabine with Rho-MRTF-SRF inhibitors could be a promising therapeutic approach for hematological malignancies.

\section{Discussion}

Using intravital BM imaging, we showed that high-dose cytarabine treatment suppressed the migration of C1498 murine AML cells, consistent with a previous study using an MLL-AF9 AML mouse model [7]. Additionally, we demonstrated that proliferative $\mathrm{C} 1498$ AML cells regain their migration abilities after chemotherapy. Reduction of cell motility in residual AML cells upon chemotherapy could be explained by two ways: (i) chemotherapy reduced the motility of residual AML cells, and (ii) less motile AML cells were chemoresistant and could survive. Although we could not exclude the possibility of the latter case (ii), we think the former explanation (i) would be more likely because the number of "less motile" residual cells after chemotherapy was much larger than that of less motile cells inherently in control conditions. Several studies suggested an important role of the BM microenvironment in the development of chemoresistance in AML [6, $12,23,24]$. Consistently, our findings suggest that extensive interactions occur between AML cells and BM microenvironment components during chemotherapy, which can promote the development of chemoresistance in residual AML cells. Osteoblasts, endothelial cells, and stromal cells are among the cell types in the BM niche that have been demonstrated to contribute to the development of chemoresistance in AML [25]. Nevertheless, a recent study involving in vivo imaging of the BM reported that the role of stromal cells in AML chemoresistance was negligible [7]. In this study, we found that the localization of AML cells within the BM did not influence the development of chemoresistance. Hence, the role of osteoblasts, endothelial cells, and stromal cells in the development of chemoresistance in AML remains unclear. Future studies involving the simultaneous tracking of various cell types are required to dissect the complex interplay between the components of the BM microenvironment and AML cells in vivo.

\section{Conclusions}

Here, we demonstrated that the Rho-MRTF-SRF signaling pathway was strongly upregulated in chemoresistant AML cells. Rho-MRTF-SRF pathway activation enhances cancer cell motility in solid tumors, leading to cancer cell migration and metastasis. MRTF-SRF pathway inhibition using CCG-1423 or its derivative CCG-203971 exerted anticancer effects in metastatic malignant melanoma and breast cancer [19, 21]. Our imaging and transcriptomic findings showed that, in contrast to solid tumors, chemotherapy suppressed AML cell migration, as well as provided novel insight into the role of the Rho-MRTF-SRF pathway in the migration of chemoresistant AML cells. 
Nevertheless, similar to solid tumors, Rho-MRTF-SRF inhibition by CCG treatment augmented the anti-tumor effects of chemotherapy in our AML mouse model. Our findings on the relationship between cell motility and AML chemoresistance provide a strong rationale for the modulation of cellular motility as a therapeutic target for refractory AML.

\section{Supplementary information}

Supplementary information accompanies this paper at https://doi.org/10. 1186/s41232-020-00127-6.

Additional file 1: Video 1. Intravital time-lapse imaging during different phases of progression of AML. AML cells were transplanted into wild-type C57BI6 mice. AML mice were subjected to intravital time-lapse BM imaging during different disease phases. Green, EGFP-expressing AML cells; red, blood vessels (Alexa Fluor 594); blue, bone tissues (SHG). Scale bar, $50 \mu \mathrm{m}$.

Additional file 2: Video 2. AML cell motility during chemotherapy. AML cells were injected into wild-type C57Bl6 mice. At days 19 and 20, the mice were treated with PBS or cytarabine. Intravital time-lapse imaging experiments were performed on day 21 (PBS control and cytarabine-treated mice) or day 28 (cytarabine-treated mice). Green, EGFP-expressing AML cells; red, blood vessels (Alexa Fluor 594); blue, bone tissues (SHG). Scale bar, $50 \mu \mathrm{m}$.

Additional file 3: Video 3. CCG treatment induces dynamic changes in chemoresistant AML cells. AML-transplanted mice were treated with CCG before/during chemotherapy and after chemotherapy, followed by intravital time-lapse BM imaging. Green, EGFP-expressing AML cells; red, blood vessels (Alexa Fluor 594); blue, bone tissues (SHG). Scale bar, 50 $\mu \mathrm{m}$

Additional file 4: Supplementary Figure 1. Intravital two-photon imaging at different phases of progression. Representative intravital twophoton MIP skull images from C57Bl6 mice 10 days (left), 18 days (middle), and 27 days (right) after AML cell injection (day 0). Green, EGFPexpressing AML cells; red, blood vessels (Alexa Fluor 594); blue, bone tissues (SHG). Scale bar, $50 \mu \mathrm{m}$. See also Video 1.

Additional file 5: Supplementary Figure 2. Survival of cytarabinetreated AML-recipient mice. (A) Kaplan-Meier plot showing survival of AML-injected mice treated with PBS or $200 \mathrm{mg} / \mathrm{kg}$ of cytarabine. (PBS, n =5; cytarabine, $\mathrm{n}=5$, from a single experiment). ${ }^{*} \mathrm{P}<0.0332$ (log-rank test). (B) Scatter plots showing the cell number of AML cells included in 10,000 of BM cells. Data are presented as mean \pm SD. ${ }^{*} P<0.05$ (Kolmogorov-Smirnov test).

Additional file 6: Supplementary Figure 3. Activation of SRF-MRTFA pathway after chemotherapy. (A) Top biological functions of the differentially expressed genes between chemotherapy-treated and control AMLrecipient mice. (B) Heatmap showing differential expression genes after cytarabine treatment, as well as diseases and biological functions they are involved in. (C) Top five master regulators determined by causal network analysis.

Additional file 7: Supplementary Figure 4. Localization of AML cells in the BM after CCG treatment. (A, C) Distribution of distance between AML cells and the bone surface (B) or blood vessels (D) after CCG treatment. Pooled data from three mice per condition from independent experiments are shown. - CCG, $n=250 ;+C C G, n=130$. (B, D) Mean distance between AML cells and the bone surface (B) or blood vessels (D). NS, not significant (Kolmogorov-Smirnov test).

\section{Abbreviations}

AML: Acute myeloid leukemia; ANOVA: Analysis of variance; BM: Bone marrow; FBS: Fetal bovine serum; GEO: Gene expression omnibus; IPA: Ingenuity pathway analysis; IoU: Intersection over union; MIP: Maximum intensity projections; MKL: Megakaryoblastic leukemia; MRTF: Myocardin- related transcription factors; PBS: Phosphate-buffered saline; SHG: Secondharmonic generation; SRF: Serum response factor

\section{Acknowledgements}

Not applicable

\section{Authors' contributions}

E.Y. and M.I. conceived the study. M.M. E.Y. and H.M. performed the in vivo imaging and analysis of AML recipient mice with the assistance of S.S., D.O., and D.M. performed the RNA-seq analysis. E.Y. wrote the initial draft. T.S., J.K., and M.I. revised the final draft. All authors approved the manuscript to be published, and agree to be accountable for all aspects of the work in ensuring that questions related to the accuracy or integrity of any part of the work are appropriately investigated and resolve.

\section{Funding}

This work was supported by funding from CREST, Japan Science and Technology Agency (to M.I.), Grants-in-Aid for Scientific Research (S) from the Japan Society for the Promotion of Science (JSPS to M.I.), a Grant-in-Aid for JSPS Fellows (to E.Y.), and grants from the Uehara Memorial Foundation (to M.I.), the Mochida Memorial Foundation (to M.I.), and the Takeda Science Foundation (to M.I.)

\section{Availability of data and materials}

The authors confirm that the data supporting the findings of this study are available within the article or its supplementary materials. Raw data were generated at Osaka University. Access to raw data concerning this study was submitted under Gene Expression Omnibus (GEO) accession number GSE149853. Derived data supporting the findings of this study are available from the corresponding author E.Y. and M.I. on request.

Ethics approval and consent to participate

The Institutional Animal Care and Use Committee of Osaka University approved all procedures involving animals.

\section{Consent for publication}

Not applicable

\section{Competing interests}

The authors declare that they have no competing interests.

The English in this document has been checked by at least two professional editors, both native speakers of English. For a certificate, please see: http:// www.textcheck.com/certificate/gYpf4F

\section{Author details}

1Department of Immunology and Cell Biology, Graduate School of Medicine and Frontier Biosciences, Osaka University, Osaka, Japan. ${ }^{2}$ WPI-Immunology Frontier Research Center, Osaka University, Osaka, Japan. ${ }^{3}$ Laboratory of Bioimaging and Drug Discovery, National Institutes of Biomedical Innovation, Health and Nutrition, Osaka, Japan. ${ }^{4}$ Department of Bioinformatic Engineering, Graduate School of Information Science and Technology, Osaka University, Osaka, Japan. ${ }^{5}$ Genome Information Research Center, Research Institute for Microbial Diseases, Osaka University, Osaka, Japan.

Received: 12 May 2020 Accepted: 8 June 2020

Published online: 06 July 2020

\section{References}

1. Löwenberg B, Downing JR, Burnett A. Acute myeloid leukemia. N Engl J Med. 1999;341(14):1051-62. https://doi.org/10.1056/NEJM199909303411407 PubMed PMID: 10502596.

2. Tallman MS, Gilliland DG, Rowe JM. Drug therapy for acute myeloid leukemia. Blood. 2005;106(4):1154-1163. Epub 2005/05/03. https://doi.org/10. 1182/blood-2005-01-0178. PubMed PMID: 15870183.

3. Yin B, Kogan SC, Dickins RA, Lowe SW, Largaespada DA. Trp53 loss during in vitro selection contributes to acquired Ara-C resistance in acute myeloid leukemia. Exp Hematol. 2006;34(5):631-41. https://doi.org/10.1016/j.exphem. 2006.01.015. PubMed PMID: 16647569.

4. Döhner H, Weisdorf DJ, Bloomfield CD. Acute myeloid leukemia. N Engl J Med. 2015:373(12):1136-52. https://doi.org/10.1056/NEJMra1406184. PubMed PMID: 26376137. 
5. Ohtake S, Miyawaki S, Fujita H, Kiyoi H, Shinagawa K, Usui N, et al. Randomized study of induction therapy comparing standard-dose idarubicin with high-dose daunorubicin in adult patients with previously untreated acute myeloid leukemia: the JALSG AML201 Study. Blood. 2011; 117(8):2358-2365. Epub 2010/08/06. https://doi.org/10.1182/blood-2010-03273243. PubMed PMID: 20693429

6. Sánchez-Aguilera A, Méndez-Ferrer S. The hematopoietic stem-cell niche in health and leukemia. Cell Mol Life Sci. 2017;74(4):579-590. Epub 2016/07/19. https://doi.org/10.1007/s00018-016-2306-y. PubMed PMID: 27436341; PubMed Central PMCID: PMCPMC5272896.

7. Duarte D, Amarteifio S, Ang H, Kong IY, Ruivo N, Pruessner G, et al. Defining the in vivo characteristics of acute myeloid leukemia cells behavior by intravital imaging. Immunol Cell Biol. 2019;97(2):229-235. Epub 2018/12/13. https://doi.org/10.1111/imcb.12216. PubMed PMID: 30422351; PubMed Central PMCID: PMCPMC6446728.

8. Hawkins ED, Duarte D, Akinduro O, Khorshed RA, Passaro D, Nowicka M, et al. T-cell acute leukaemia exhibits dynamic interactions with bone marrow microenvironments. Nature. 2016;538(7626):518-522. Epub 2016/10/ 17. https://doi.org/10.1038/nature19801. PubMed PMID: 27750279; PubMed Central PMCID: PMCPMC5164929.

9. Bajaj J, Konuma T, Lytle NK, Kwon HY, Ablack JN, Cantor JM, et al. CD98mediated adhesive signaling enables the establishment and propagation of acute myelogenous leukemia. Cancer Cell. 2016;30(5):792-805. Epub 2016/ 10/27. https://doi.org/10.1016/j.ccell.2016.10.003. PubMed PMID: 27908736; PubMed Central PMCID: PMCPMC5137811.

10. Nikolova M, Guenova M, Taskov H, Dimitrova E, Staneva M. Levels of expression of CAF7 (CD98) have prognostic significance in adult acute leukemia. Leuk Res 1998;22(1):39-47. https://doi.org/10.1016/s01452126(97)00129-x. PubMed PMID: 9585078.

11. Reinisch A, Majeti R. Sticking it to the niche: CD98 mediates critical adhesive signals in AML. Cancer Cell 2016;30(5):662-664. https://doi.org/10.1016/j.ccell. 2016.10.014. PubMed PMID: 27846387

12. Lane SW, Scadden DT, Gilliland DG. The leukemic stem cell niche: current concepts and therapeutic opportunities. Blood. 2009;114(6):1150-1157. Epub 2009/04/28. https://doi.org/10.1182/blood-2009-01-202606. PubMed PMID: 19401558; PubMed Central PMCID: PMCPMC2723012.

13. Duarte D, Hawkins ED, Lo Celso C. The interplay of leukemia cells and the bone marrow microenvironment. Blood. 2018;131(14):1507-1511. Epub 2018/02/27. https://doi.org/10.1182/blood-2017-12-784132. PubMed PMID: 29487069.

14. Ishii M, Egen JG, Klauschen F, Meier-Schellersheim M, Saeki Y, Vacher J, et al. Sphingosine-1-phosphate mobilizes osteoclast precursors and regulates bone homeostasis. Nature. 2009;458(7237):524-528. Epub 2009/02/08. https://doi.org/10.1038/nature07713. PubMed PMID: 19204730; PubMed Central PMCID: PMCPMC2785034

15. Ishii M, Kikuta J, Shimazu Y, Meier-Schellersheim M, Germain RN. Chemorepulsion by blood S1P regulates osteoclast precursor mobilization and bone remodeling in vivo. J Exp Med. 2010;207(13):2793-2798. Epub 2010/12/06. https://doi.org/10.1084/jem.20101474. PubMed PMID: 21135136; PubMed Central PMCID: PMCPMC3005230.

16. Kikuta J, Wada Y, Kowada T, Wang Z, Sun-Wada GH, Nishiyama I, et al. Dynamic visualization of RANKL and Th17-mediated osteoclast function. J Clin Invest. 2013;123(2):866-873. Epub 2013/01/16. https://doi.org/10.1172/ JCl65054. PubMed PMID: 23321670; PubMed Central PMCID: PMCPMC3561830.

17. Kikuta J, Kawamura S, Okiji F, Shirazaki M, Sakai S, Saito H, et al. Sphingosine-1-phosphate-mediated osteoclast precursor monocyte migration is a critical point of control in antibone-resorptive action of active vitamin D. Proc Natl Acad Sci U S A. 2013;110(17):7009-7013. Epub 2013/04/ 08. https://doi.org/10.1073/pnas.1218799110. PubMed PMID: 23569273; PubMed Central PMCID: PMCPMC3637769.

18. Medjkane S, Perez-Sanchez C, Gaggioli C, Sahai E, Treisman R. Myocardinrelated transcription factors and SRF are required for cytoskeletal dynamics and experimental metastasis. Nat Cell Biol. 2009;11(3):257-268. Epub 2009/ 02/08. https://doi.org/10.1038/ncb1833. PubMed PMID: 19198601; PubMed Central PMCID: PMCPMC6089348.

19. Whitson RJ, Lee A, Urman NM, Mirza A, Yao CY, Brown AS, et al. Noncanonical hedgehog pathway activation through SRF-MKL1 promotes drug resistance in basal cell carcinomas. Nat Med. 2018;24(3):271-281. Epub 2018/02/05. https://doi.org/10.1038/nm.4476. PubMed PMID: 29400712; PubMed Central PMCID: PMCPMC5839965.
20. Miralles F, Posern G, Zaromytidou Al, Treisman R. Actin dynamics control SRF activity by regulation of its coactivator MAL. Cell. 2003;113(3):329-342. https://doi.org/10.1016/s0092-8674(03)00278-2. PubMed PMID: 12732141.

21. Bell JL, Haak AJ, Wade SM, Kirchhoff PD, Neubig RR, Larsen SD. Optimization of novel nipecotic bis(amide) inhibitors of the Rho/MKL1/SRF transcriptional pathway as potential anti-metastasis agents. Bioorg Med Chem Lett. 2013; 23(13):3826-3832. Epub 2013/05/07. https://doi.org/10.1016/j.bmcl.2013.04. 080. PubMed PMID: 23707258; PubMed Central PMCID: PMCPMC3707396.

22. Gau D, Veon W, Capasso TL, Bottcher R, Shroff S, Roman BL, et al. Pharmacological intervention of MKL/SRF signaling by CCG-1423 impedes endothelial cell migration and angiogenesis. Angiogenesis. 2017;20(4):663672. Epub 2017/06/21. https://doi.org/10.1007/s10456-017-9560-y. PubMed PMID: 28638990; PubMed Central PMCID: PMCPMC5985144.

23. Ishikawa F, Yoshida S, Saito Y, Hijikata A, Kitamura H, Tanaka S, et al. Chemotherapy-resistant human AML stem cells home to and engraft within the bone-marrow endosteal region. Nat Biotechnol 2007;25(11):1315-1321. Epub 2007/10/21. https://doi.org/10.1038/nbt1350. PubMed PMID: 17952057.

24. Zhou HS, Carter BZ, Andreeff M. Bone marrow niche-mediated survival of leukemia stem cells in acute myeloid leukemia: Yin and Yang. Cancer Biol Med. 2016;13(2):248-259. https://doi.org/10.20892/j.issn.2095-3941.2016.0023. PubMed PMID: 27458532; PubMed Central PMCID: PMCPMC4944541.

25. Duarte D, Hawkins ED, Akinduro O, Ang H, De Filippo K, Kong IY, et al. Inhibition of endosteal vascular niche remodeling rescues hematopoietic stem cell loss in AML. Cell Stem Cell. 2018;22(1):64-77.e6. Epub 2017/12/21. https://doi.org/10.1016/j.stem.2017.11.006. PubMed PMID: 29276143. PubMed Central PMCID: PMCPMC5766835.

\section{Publisher's Note}

Springer Nature remains neutral with regard to jurisdictional claims in published maps and institutional affiliations.

\section{Ready to submit your research? Choose BMC and benefit from:}

- fast, convenient online submission

- thorough peer review by experienced researchers in your field

- rapid publication on acceptance

- support for research data, including large and complex data types

- gold Open Access which fosters wider collaboration and increased citations

- maximum visibility for your research: over 100M website views per year

At BMC, research is always in progress.

Learn more biomedcentral.com/submissions 